\title{
I volgari municipali e l'affioramento di una scripta nel medioevo lombardo
}

\author{
Giuseppe Polimeni
}

\begin{abstract}
L'articolo si propone di motivare come nella storia linguistica delle città lombarde lingual dialetto non siano sistemi contrapposti, ma registri strettamente legati. Le indicazioni manzoniane in apertura invitano a osservare gli statuti cittadini del medioevo come luogo di contatto e di scambio tra due sistemi linguistici, latino e volgare.

Senza varcare di proposito la soglia di alcuni dei più antichi manoscritti lombardi, l'articolo cerca di rilevare nelle testimonianze dell'originaria diglossia una ben più complessa sintonia di affioramenti e di successivi strappi, osservando nella lente della scrittura un passaggio di testimone, quello tra latino e volgare, che le opposizioni scolastiche al pari dei facili fraintendimenti terminologici non aiutano a sciogliere.
\end{abstract}

Parole chiave: latino medievale, rete della grafia, uso lessicale specifico, uso «riflesso», ricezione e circolazione di lingue.

\section{Abstract}

The article aims at assessing the motives for which, in the linguistic history of the Lombardian cities, lingua/dialetto (language/dialect) are not contraposed systems, but are instead highly interlinked registers. The Manzonian information given at the beginning of the article invites us to consider the medieval city statutes as a point of contact and exchange between two linguistic systems, Latin and the vulgate.

Without expressly crossing the threshold of some of the oldest Lombardian manuscripts, the article attempts to underline, within the evidence provided by the original digloss, a complex awareness both of growth and of successive breakings, observing, through the lens of writing, a passage of testimony between Latin and the vulgate, which scholastic opposition, just as with facile terminological misunderstandings, does not help to clarify.

Key words: Medieval Latin, graphic network, specific lexical use, reflexive use, reception and circulation of languages.

Nel cuore di Milano, all'angolo tra via Morone e piazza Belgioioso, Alessandro Manzoni concludeva nell'ottobre 1843 il primo capitolo del libro Della lingua italiana. Postillatore attento del dizionario di Cherubini e lettore partecipe del Porta, non poteva sfuggirgli che «una lingua non è altro che un mezzo d'in- 
tendersi uomini con uomini» ${ }^{1}$ e che di conseguenza, anche quando il lapsus calami sembra per un attimo mescolare le carte in tavola, "tra quelli che si chiamano dialetti, e quelle che si chiamano lingue» corre "una differenza reale». ${ }^{2}$

[...] se dicessimo che, essendoci in Italia una quantità d'idiomi più o meno diversi, s'ha bisogno d'una lingua comune, per non esser ridotti, o a non aver che con pochi una agevole, piena, sicura comunion di linguaggio, o a spender la vita in imparar linguaggi; se dicessimo che questo è l'unico mezzo per noi di mettere insieme il lavoro di molte intelligenze, di partecipare immediatamente tutti del lavoro d'alcuni; che da questo avere o non avere una lingua comune dipende per noi l'essere una nazione, o una moltitudine di tribù, l'esser riuniti in una civilizzazione comune, o divisi in non so quante barbarie; troverebbero stravaganza il creder necessario di dir cose tali, ingiuria il dirle a loro. $^{3}$

Dai precordi della cultura milanese, che è del Maggi e di Parini come dei Verri e di Beccaria, era germogliata e approdava a formulazione teorica la «ricerca» di uno strumento comunicativo e espressivo che rispondesse alle esigenze di un'intera società. La scelta del toscano prima e, dall'Ottocento, dell'italiano, «lingue di cultura» in apparente pressione sull'idioma "di natura», scaturisce da una storia e da una riflessione che fatti diversi concorrono a definire cittadine ed europee.

Così l'avvicinamento a un ideale di lingua nazionale e sovranazionale può trovare un'utile chiave di lettura proprio nelle vicende della cultura dialettale di una regione, che verso quell'ideale è chiamata a convergere perché, fin dalle origini, mosaico variegato di punti e di identità linguistiche.

Una pluralità di parlate, $\mathrm{i}$ cui confini corrono sulle mura delle singole città $\mathrm{e}$ lungo i corsi d'acqua del contado, Manzoni ascoltava nella «latinità babilonesca» trasmessa dagli statuti «scritti nella seconda metà, dirò così, del medio evo".

Il «guazzabuglio» dei linguaggi pareva infatti già ben definito e pericoloso là dove si trattava «di prescrivere, di proibire, di permettere, di regolar le azioni e le relazioni degli uomini». ${ }^{4}$ A chi aveva "un po' frugato nelle opere buone e triste dei varii tempi della letteratura italiana",

1. Alessandro Manzoni, Scritti linguistici inediti, a cura di Angelo STella e Maurizio VitaLE, premessa di Giovanni NeNCIONI, Milano: Centro nazionale Studi manzoniani, 2000, vol. XVII dell'Edizione nazionale ed europea delle opere di Alessandro Manzoni, t. I, p. 345.

2. «[...] bisognerebbe esaminare se i dialetti siano o non siano lingue; o in altri termini, se tra quelli che si chiamano dialetti, e quelle che si chiamano lingue ci sia una differenza reale, e se consista nell'essenza della cosa, o sia puramente accidentale», ibid., p. 355-356; "[la lingua francese] Non ha, come noi, un'altra lingua... oh! scusate: m'è scappata questa parola, non rammentandomi che è un punto in questione se il milanese e gli altri che chiamiam dialetti, siano o non siano lingue; non ha, dirò dunque, come noi, un altro mezzo col quale nomini quelle cose, e le senta nominare», ibid., p. 365.

3. Ibid., p. 343-344.

4. Ibid., p. 374.

5. Alessandro MANZONI, Seconda "Introduzione» al "Fermo e Lucia», in Scritti linguistici inediti, cit., t. I, p. 21, $\$ 17$. 
tro offrivano l'occasione per osservare le differenze e le peculiarità, che inducevano a parlare di «latino dunque, ma latino di Milano [...] latino di Modena [...] latino di Genova [...] latino di Bergamo [...] latino di Firenze [...] latino di Parma», ${ }^{6}$ segnalando poi lo specifico lessicale dei documenti di Venezia, Ferrara, Torino, Napoli, Pavia, Bologna, Lodi e Cremona, Padova e Feltre, Ivrea, Novara.

Suggerito, agli albori di una storia linguistica nazionale, il dialogo sotterraneo che, tra latino e volgare, ha messo in atto per secoli le convergenze di una diplomazia non ufficiale, l'argomentazione manzoniana invita a leggere sotto l'apparente frizione tra gli idiomi una scala di registri e di situazioni comunicative, su cui dal Cinquecento - e con più chiarezza nel teatro - si disporranno gradatamente toscano/italiano e dialetto. L'attribuzione dei ruoli e la scelta dei propositi, spesso apertamente dichiarati ma non altrettanto sistematicamente perseguiti, trovano forse origine nella percezione di un'identità grafica e lessicale, quando non anche fonetica, che ha le sue basi nella scripta latina e poi volgare del medioevo.

Sulla traccia indicata dalla riflessione di Manzoni e cinquant'anni dopo dalla ricerca dialettologica di Carlo Salvioni, ${ }^{7}$ questo lavoro cerca più modestamente di considerare alcuni problemi teorici emersi dall'analisi della grafia dei documenti latini del tardo medioevo e dei sincroni testi volgari, dove è dato di misurare l'adesione progressiva a un codice, in primo luogo grafico, comune.

Senza varcare di proposito la soglia di alcuni dei più antichi manoscritti lombardi, il tentativo di rilevare nelle testimonianze dell'originaria diglossia una ben più complessa sintonia di affioramenti e di successivi strappi vuole prefigurare la direzione futura del contatto e dello scambio tra idiomi, osservando nella lente della scrittura un passaggio di testimone che le opposizioni scolastiche al pari dei facili fraintendimenti terminologici evitano di sciogliere.

Proprio il latino medievale, con la sua superficiale omogeneità e unità normalizzante, è oggi testimonianza superstite della specificità linguistica dei singoli centri nell'area che ci proponiamo di considerare, ma è soprattutto indicazione di una tappa in cui il volgare gradualmente cerca codificazione, oltre che attestazione scritta, fino a ritagliarsi anche attraverso la grafia usi e destinatari propri. ${ }^{8}$

\section{Ibid., p. 374-379.}

7. Carlo SALVIONI, "L'elemento volgare negli statuti latini di Brissago, Intragna e Malesco», in Bollettino storico della Svizzera Italiana, XIX, 1897, p. 133-170.

8. Nel Trecento, là dove il volgare ha raggiunto piena maturità grafica, il latino lascia il campo a un nuovo codice scritto, in cui trova più trasparente codificazione, giuridica e linguistica, la vita quotidiana; cfr. Gli statuti delle strade e delle acque del contado di Milano, a cura di Angelo STELla e di Luciano F. FARINA, con la collaborazione di Giovanna BALESTRERI, contributi di Pierluigi TozzI e Massimiliano DAVID, Milano: LED, 1992. Non solo un mutamento di destinatario, ma un contesto politico diverso animano l'adozione del volgare presso la cancelleria visconteo-sforzesca: cfr. Maurizio VITALE, La lingua volgare della Cancelleria Visconteo-Sforzesca nel Quattrocento, con una premessa di Antonio VISCARDI, Milano: Cisalpino, 1953. 
Nel documento notarile, come nello statuto, la parte fissa, e comune a una società dalle esigenze diversamente globali, pare a tutti gli effetti senza tempo, predisposta ad assegnare l'evento e i protagonisti a quell' eternità «non corruttibile», che il latino soltanto, come Dante anticipava nel Convivio, ${ }^{9}$ poteva garantire.

Là dove invece le cose e il mondo materiale si affacciano alla pagina dello statuto o dell'atto, il vetro della "gramatica» si increspa e lascia leggere preziose testimonianze della lingua d'uso, quella con cui nella quotidianità si nominano i luoghi e gli oggetti e che, come tale, va citata con precisione, anche quando sembra contraddire la morfologia della lingua ospite. Era questa la parte mobile, sensibile del documento, volta per volta adattata da notai di cultura diversa, e all'occorrenza letta e tradotta per i contraenti o per i loro eredi. ${ }^{10}$

Insieme al lessico, riporta a un'area precisa la fonetica, quella che affiora dalle smagliature grafiche dell'imprecisione o da una correzione che aggiorna e normalizza la forma di un toponimo caduta per errore o per abitudine dalla penna di un funzionario meno sorvegliato.

Il 30 settembre 1098, Otto notarius, redigendo lo «strumento» con cui Ermengarda, badessa del monastero di San Salvatore e Santa Giulia di Brescia, cede due sorti a Walcoso e Pietro, figlio del fu Giovanni di Montegalda, attua una correzione singolare, ma indicativa:

[...] datis et consignatis predictis dennariis et cera per iam dictos Walcosus et Petrus suosque heredes vel eorum miso in suprascripto monasterio Sanctae Iurie (? Iulie) ad suprascriptam abbatisam vel eius successori aut suo miso allia superi(m)posita eis non fiad. ${ }^{11}$

9. Dante Alighieri, Convivio, in Opere minori, a cura di Cesare Vasoli e Domenico De RoberTIS, Milano-Napoli: Ricciardi, 1988, t. I, parte II, p. 33-34: «Per nobilità, perché lo latino è perpetuo e non corruttibile, e lo volgare è non stabile e corruttibile. Onde vedemo ne le scritture antiche de le comedie e tragedie latine, che non si possono transmutare, quello medesimo che oggi avemo; che non avviene del volgare, lo quale a piacimento artificiato si transmuta. Onde vedemo ne le cittadi d'Italia, se bene volemo agguardare, da cinquanta anni in qua molti vocabuli essere spenti e nati e variati; onde se 'l picciol tempo così transmuta, molto più transmuta lo maggiore. Sì ch'io dico, che se coloro che partiron d'esta vita già sono mille anni tornassero a le loro cittadi, crederebbero la loro cittade essere occupata da gente strana, per la lingua da loro discordante»; sul mutamento diatopico e diacronico cui è soggetto il volgare si veda De vulgari eloquentia I, IX, 4-11 e il rimando, individuato da Maria Corti, a Restoro D'ArEZZO, La composizione del mondo, a cura di Alberto Morino, Milano-Parma: Fondazione Pietro Bembo-Ugo Guanda, 1997, II.7.4 (24-25), p. 294-295.

10. Valgono per i documenti tardomedievali le indicazioni che Francesco Sabatini ricava per la «tradizione di lingua scritta intermedia» dell'alto medioevo: Francesco SABATINI, «Dalla «scripta latina rustica» alle «scriptae» romanze», in ID., Italia linguistica delle origini, saggi editi dal 1956 al 1996 raccolti da Vittorio Coletti, Rosario ColuCCiA, Paolo D’ACHILle, Nicola De Blasi, Livio Petrucci, Lecce: Argo, 1996, p. 119-265, articolo apparso in Studi medievali, s. III, IX, 1968, p. 320-358; in particolare si segnala, alle p. 222-225, il riferimento ai canali indiretti individuati da Lüdtke: Protokoll («registrazione scritta di un discorso orale») e Vorlesen ("presentazione orale di un testo scritto»); cfr. Helmut LÜDTKE, «Die Entstehung romanischer Schriftsprachen», in Vox Romanica, XXIII, 1964, p. 3-21.

11. Diana VeCCHIO, Le pergamene del Codice Diplomatico Bresciano (secc. XI-XII): Carte private, comunali, vescovili. Saggio di edizione, Tesi di laurea in Paleografia, Relatore Antonio 
Con l'incerta concordanza e articolazione dei casi latini, il notaio affidava alla pergamena la testimonianza di una fase arcaica della lingua, di cui non restano per l'area bresciana attestazioni nei documenti volgari: l'ipotesi, del resto sin qui non altrimenti dimostrabile, che il rotacismo potesse con ogni probabilità essere attestato in origine anche di là dell'Adda, ${ }^{12}$ è utile se non altro a ricordarci quell'originaria unità e distribuzione di fenomeni fono-morfologici, le cui tracce superstiti Carlo Salvioni aveva ritrovato nell'area della Lombardia medievale. ${ }^{13}$

Anche su altri versanti i documenti notarili riservano indicazioni significative di una lingua che, muovendo i primi passi sulla strada della maturità grafica, è ancora debitrice al latino in primo luogo della grammatica: l'antico plurale in -ora, che avrà sporadiche sopravvivenze settentrionali, ${ }^{14}$ resta ad esempio nelle maglie dalle carte bresciane nel toponimo Campora, località sita nel territorio di Nuvolera, ${ }^{15}$ come nella consuetudine testimoniata dal discorso diretto di Lanfrancus iudex, dove tra le proprietà del monastero di Santa Giulia di Brescia si annoverano "vites et camporas que regitur per Laurenzo masario». ${ }^{16}$

Indizi preziosi vengono, come si vede, dai toponimi e in genere dall'onomastica, che in forme ormai pressoché cristallizzate permette, se non di ricostruire, almeno di ipotizzare una situazione anteriore a quella che si riscontra nei manoscritti trecenteschi in volgare. ${ }^{17}$ Le pergamene del Codice Diplomatico Bresciano aprono inoltre un'altra finestra sulla nascita dei cognomi: accanto ai patronimici e all'indicazione di provenienza, si registrano tra XII e XIII secolo professioni («Teudoldus qui dicitur Malgarita atque etiam Mauruntus qui dicitur Canevarius», ${ }^{18}$ "Girbellinus Pistor de Leno») ${ }^{19}$ e soprannomi, raramente purgati della loro marca di oralità (Oddo qui dicitur Lisignolus, Andreottus Cagapistus, Guaço Pesacarnem, magistrum Albertum Quatercapellum, pre' Sechafeno, dominus Lanfrancus Buccadelucius).

Nella descrizione di luoghi e cose, come detto, il ductus latino è chiamato a ospitare il parlato, con formule che rispecchiano non solo il nome proprio, ma anche la disposizione di persone e oggetti: quella spaziale («da una parte

Ciaralli, Correlatore Ezio BARbIERI, Università degli Studi di Verona, Facoltà di Lettere e Filosofia, a.a. 2001-2002, p. 30-31; atto rogato a Brescia il 30 settembre 1098, dove appunto - $l$ - risulta corretta su $-r$ -

12. Il rotacismo, ampiamente testimoniato nei coevi testi pavesi-milanesi dell'abbazia di Morimondo, sarà localizzato nel secolo successivo in un'area che ha come vertice Bellinzona e per base la Liguria: cfr. Angelo Stella, Lombardia, in Luca Serianni e Pietro Trifone (a cura di), Storia della lingua italiana, vol. III, Le altre lingue, Torino: Einaudi, 1994, p. 153-212.

13. Carlo Salvioni, Ladinia e Italia, Milano: Tipografia Padoan, 1938, p. 19-29.

14. Cfr. Gerhard RoHLfs, Toponomastica italiana (origini, aspetti e problemi), in ID., Studi e ricerche su lingua e dialetti d'Italia, Firenze: Sansoni, 1972, p. 52.

15. Diana VECCHIO, Le pergamene..., cit., p. 8; atto rogato il 31 maggio 1041.

16. Ibid., p. 13; atto rogato il 26 maggio 1050.

17. Sull'apporto della toponomastica allo studio storico del volgare, con riscontro sulle voci e sulle sopravvivenze municipali, cfr. Angelo STELLA, Lombardia, cit., p. 154-155.

18. Diana VECCHIO, Le pergamene..., cit., p. 38; atto rogato il 20 maggio 1126.

19. Ibid., p. 123; atto rogato il 17 ottobre 1194. 
Petro cum suis consortes, da alia parte Burningus», ${ }^{20}$ «in curte maiore subtus laubia frascata») ${ }^{21}$ come quella temporale ("de hinc in antea»). ${ }^{22}$ I casi latini e l'incertezza in una morfologia non più dell'uso possono forse nascondere insidie per la chiarezza dell'atto notarile; ecco allora entrare in gioco l'affermata consuetudine preposizionale del volgare, a definire "pecias de terra vidates", "peciam de terra aratoria", "pecia una de terra in parte aratoria, in parte bosco".

Se la veste grafica è quella offerta, più che imposta, dal latino, in luoghi diversi i documenti presentano impercettibili variazioni, che torneranno utili alla lettura dei volgari, ${ }^{23}$ sia sul versante più complesso dei digrammi (acolochus, Pachana) che nelle forme destinate a persistere sin dentro i più antichi codici di Bonvesin de la Riva: si pensi a $k$ per l'occlusiva velare sorda già nella seconda metà dell' XI secolo e poi con maggiore frequenza dalla fine del XII ( karitati vestre», ${ }^{24}$ "Iacobus Rogerii Franceski», ${ }^{25}$ "Markisius Ballius», ${ }^{26}$ "de Kerbo" ${ }^{27}$ ), a $x$ per il fonema sibilante sonoro (trex, quadrageximo, Dixentiano, Angoxola, Turrexella), e si rilevino nel Codice Diplomatico Bresciano le prime occorrenze del digramma -th- per la dentale ormai in fase di lenizione, con netto valore fricativo (Carpenetholo, Prathal, Patherno, Cavethani), che Gianfranco Contini avrebbe riscontrato negli antichi testi bresciani. ${ }^{28} \mathrm{Da}$ segnalare a proposito l'oscillazione grafica nella resa del tratto tenue delle dentali finali (fiad, licead, placuid accanto a quicquit, aliquit), a dimostrazione dell'avvenuto processo di indebolimento e delle ormai diffuse forme di correzione. ${ }^{29}$

Con gradualità, ma solo a partire dall'ultimo decennio del XII secolo, si fa strada nei documenti notarili il grafema ç per l'affricata dentale (dominus Geço de Albertus Çuche, Iohaninus filius Iohannis de Çelario, Iohanni de Peçardo), proprio negli anni in cui è dato di registrare sulla scala diastratica l'oscillazione tra palatale e dentale (Sancti Genesii / Sancti Zenesii, ${ }^{30}$ ma anche Caciago / Cazago ${ }^{31}$ ).

Nella parte «intermedia» del documento, dove l'eternità del latino e dell'archivio si misura con la mutevolezza del volgare e della vita, anche la grafia

20. Ibid., p. 2; atto rogato a Brescia il 9 marzo 1015.

21. Ibid., p. 12; atto rogato il 26 maggio 1050.

22. Ibid., p. 44; atto rogato l'11 aprile 1134 .

23. A proposito degli statuti di Brissago, Intragna e Malesco, datati tra XIII e XVI secolo, Salvioni fa rilevare che gli "spedienti» grafici «sono gli stessi che s'incontrano ne' testi contemporanei di volgare lombardo" (Carlo SALVIONI, "L'elemento volgare...", cit., p. 135).

24. Diana VECCHIO, Le pergamene..., cit., p. 106; atto rogato con ogni probabilità nel 1189.

25. Ibid., p. 113; atto rogato il 4 novembre 1191.

26. Ibid., p. 123; atto rogato il 17 ottobre 1194.

27. Ibid., p. 151; atto rogato il 16 maggio 1196.

28. Angelo STElla, Lombardia, cit., p. 170-174 (Indizi per un canone del bresciano trecentesco). Cfr. inoltre Giuseppe Bonelli, Gianfranco ConTINI (a cura di), "Antichi testi bresciani», in L'Italia dialettale, XI, 1935, p. 115-152; sulla grafia -th-si vedano p. 139 e p. 146.

29. Diana VeCCHIO, Le pergamene..., cit., p. 1-2: atto rogato il 9 marzo 1015; cfr. anche Giuseppe Bonelli, Gianfranco ConTINI (a cura di), "Antichi testi bresciani», cit., p. 146.

30. Diana VeCCHIO, Le pergamene..., cit., p. 139; atto rogato tra il 17 ottobre 1194 e il 9 febbraio 1195; per il fenomeno Giuseppe Bonell, Gianfranco ConTINI (a cura di), "Antichi testi bresciani", cit., p. 144.

31. Diana VeCCHIO, Le pergamene..., cit., p. 125; atto rogato il 17 ottobre 1194. 
diventa strumento sensibile per assegnare voce inconfondibile alle cose e ai luoghi: nel sintagma "ecclesie Sancti Desciderii» ${ }^{32}$ un «nuovo» digramma registra ad esempio per l'area bresciana l'intacco palatale della sibilante, così come in altre pergamene, già nel corso del XII secolo, la gramatica perde sillabe $\mathrm{e}$ fonemi atoni, segnale di una fase matura del processo di apocope vocalica distintivo del parlato settentrionale (Domafol / Domafollus, pre’ Albertus).

Certo il latino è sopravvissuto a noi come grafia, rispettando le attese che alla sua eternità affidava l'uomo medievale, ma in un bilancio steso a margine di questi rilievi è lecito domandarsi quale fosse l'effettiva lettura che il notaio avrebbe dato di quell'atto, con quale fonetica avrebbe interpretato la scrittura immortale della gramatica, cosa del suono di quel mondo non è rimasto impigliato nella rete della grafia.

Otto e i suoi colleghi bresciani, come i notai che in quel secolo operavano nelle città lombarde e italiane, offrono comunque, per cultura ma più spesso per necessità, la prima occasione storica per rilevare quella trasparenza che Manzoni segnalava, a margine del manoscritto dell'Anonimo, in più tarde carte d'archivio:

Quando l'uomo che parla abitualmente un dialetto si pone a scrivere in una lingua, il dialetto di cui egli s'è servito nelle occasioni più attive della vita, per l'espressione più immediata e spontanea dei suoi sentimenti, gli si affaccia da tutte le parti, s'attacca alle sue idee, se ne impadronisce, anzi talvolta gli somministra le idee in una formola; gli cola dalla penna e se egli non ha fatto uno studio particolare della lingua, farà il fondo del suo scritto. ${ }^{33}$

La possibilità di «argomentare» dallo stile «la patria dello scrittore» è indicazione che va estesa, come si è visto, anche ai documenti latini del Medioevo, che interrogati e confrontati sistematicamente su più vasto raggio, aiuterebbero a ritrovare o a tracciare le singole isoglosse fino a ipotizzare il movimento dei confini di ciascuna area e a chiarire il rapporto che, proprio dalla tarda età di mezzo, lega i centri di irradiazione alla periferia.

Lembi estremi, per molti aspetti più conservativi, dell'area lombarda si dimostrerebbero le isole linguistiche, che all'interno di sistemi diversi hanno conosciuto un'evoluzione indipendente dell'originaria parlata: si pensi ai lombardi di Sicilia, che, giunti a Corleone e nell' isola in diverse ondate migratorie tra la metà del Due e il Quattrocento, chiedono a più riprese di vivere «more Tusce et Lombardie». La vicenda storica degli «oppida Lombardorum» è oggi riportata alla luce dal lavoro del gruppo di ricerca coordinato da Ezio Barbieri, ${ }^{34}$ attraverso l'edizione dei documenti dell'Archivio di Stato di Palermo, sezione di S. Maria

32. Ibid., p. 149; 4 marzo 1195; cfr. anche «sine conscilio», p. 145; atto rogato tra il 17 ottobre 1194 e il 9 febbraio 1195 .

33. Alessandro MANZONI, Seconda «Introduzione» al "Fermo e Lucia», cit., p. 21, \$14.

34. Ezio BARBIERI, «Lombardi in Sicilia. Osservazioni diplomatistiche sui loro documenti (secc. XIII-XVI)», in Marzio DALL'ACQUA (a cura di), Documenta '93, Parma: Archivio di StatoScuola di Archivistica, Paleografia e Diplomatica, biennio 1991-1993, p. 73-83; si fa riferimento ai documenti notarili del monastero di S. Maria del Bosco di Calatamauro. 
della Catena: di queste generazioni periferiche, forse vere e proprie «colonie», bisognerà tenere conto, nella prospettiva già indicata anche da Salvioni, ${ }^{35}$ per riportare indicazioni sulla lingua e sulla cultura volgare della regione di origine.

Dal latino, registro delle cronache e dell'apologia colta, più ancora che dai manoscritti in volgare, scaturisce per tutto il Duecento anche l'immagine della città come centro di localizzazione di una forma linguistica in grado di identificare una comunità di parlanti: la gramatica fa spazio con nuova consapevolezza a tessere lessicali dell'uso e attribuisce alla parola il merito di unire e distinguere, riconoscibile com'è a distanza, in luoghi e da parlanti diversi. ${ }^{36}$

Con la descrizione di Milano, Bonvesin de la Riva accoglie e per primo giustifica nel De magnalibus la parlata della sua civitas, annotandone con scrupolo il vocabolario dentro la morfologia latina:

Tecta vicinis platearum earum comunia, que vulgo coperta vocantur,.LX. fere numeri culmen ascendunt (II.2).

Porte quoque civitatis principales fortissime sunt, que numerum complent senarium. Secundarie vero sunt decem, que vocantur pusterle, in quibus omnibus mirabilis muri mirabile cernitur undique fundamentum [...] (II.4).

Quando fit universalis exercitus, currus publice oculis humanis mirandum spectaculum prestans, qui vulgo carrocerum dicitur, scarlatu circumquaque opertus et decenter ornatus (v.24).

Annotazione puntuale di un uso, la cronaca, al pari del documento, registra di proposito la forma "depurata» della voce, ma a differenza dell'atto spesso ne ristabilisce la prospettiva storica, suggerendo un'interpretazione che recupera o echeggia i repertori lessicografici del medioevo:

35. Carlo SALVIONI, Ladinia e Italia, cit.; p. 27-28: «Per la Lombardia, una prova capitale viene dalla Sicilia, e ce la fornisce la storia. Sono laggiù, a S. Fratello, Piazza Armerina, Novara e in qualche altro comune, delle popolazioni che i siciliani chiamano "lombarde" attribuendo certo a questo patronimico il suo senso medievale di "italiano cisappennino", ma che noi potrem chiamare lombarde anche nel senso attuale della parola. Sono genti nostre emigrate verso l'Etna in età medievale non certa, ma anteriore senza dubbio di qualche secolo al primo apparire d'una letteratura lombarda. Parlano un linguaggio gallo-italico, cioè "lombardo" nel senso antico, che prima, male interpretati certi passi degli storici o tratte da questi illazioni eccessive, si credeva monferrino, ma che poi, ridotta la questione, in mancanza d'altri elementi, a un puro problema linguistico, è stato identificato, soprattutto per la varietà di $\mathrm{S}$. Fratello, col dialetto lombardo, e più precisamente con quello di un settore alpino che corrisponderebbe all'alto novarese e al ticinese d'occidente. [...] Ora, S. Fratello altera la formola $c a$ secondo il vezzo latino. E se il Meyer-Lübke, quando ancora credeva all'origine monferrina dei lombardi dell'isola, non esitava a decidere che il kja di S. Fratello provava per un kja dell'antico Monferrato, collo stesso diritto sentenzieremo noi ora che esso provi per un $k j a(c a)$ lombardo molto diffuso già oltre i suoi limiti attuali».

36. BonVESIN De la RIVA, De magnalibus Mediolani, testo critico, traduzione e note a cura di Paolo CHIESA, Milano: Libri Scheiwiller, 1998, cap. III: «Sicut eorum idioma facilius alio loquitur in linguarum diversitate intelligiturque vice conversa, sic etiam inter omnes gentium (facie sola) in omnibus dinoscuntur». 
Unum tantum, etsi cetera omnia sileantur, annuntio, quoniam tam in civitate quam extra ingentis est nobilitatis quantitas numerosa virorum, quorum quidem pars magna valvasores dicuntur a valvis. Quoniam, cum Romanorum imperatores in Ambroxiano pritorio morarentur, hec erat eorum dignitas, quia erant imperatorie curie portenarii. Alii vero maioris nobilitatis capitanei nuncupantur a capite: fuerant enim plebium capita (III. 34).

La generazione successiva, per intendersi quella di Galvano Fiamma che scrive tra gli archi del convento di Sant'Eustorgio di Milano dentro le mura della cittadella viscontea, potrà attingere al vocabolario messo a punto da Bonvesin, con la certezza che esso sia ormai testato e codificato.

Alle soglie del secolo XIII ciascun cronista, nell'atto di collocare la città al centro (e per la precisione in medio) dell'universo, vuole marcare e quasi garantirne l'uso lessicale specifico, proprio appoggiandosi alla percezione contrastiva tra la lingua eterna e la lingua che muta, di punto in punto e nel tempo. ${ }^{37}$ Non diversamente da Bonvesin, ma vent'anni dopo dalla specola avignonese, Opicino de Canistris evoca, con le immagini, le parole della natia Ticinum, proposte e salvate nella lingua che sola può garantire il prestigio e la permanenza, anche nella distanza spaziale:

Docti sunt enim valde tam in aqua, quam in terra pugnare, facientes, cum necesse fuerit, in navibus machinas et in terris, ac naves acutas, cursuque veloces, quas scancerias vocant, ad pugnandum in aqua.

Cum ad solempnem et generalem procedunt exercitum, secum aliquando ducunt plaustrum trahentibus pluribus paribus boum panno rubeo coopertorum, quod plaustrum vulgo Carochium dicitur.

[...] singulis diebus dominicis atque festis et in die Carnis Privii cum quinta feria precedenti, quedam spectacula faciunt, que vulgo bataliole, set latine convenientius bellicula nuncupantur.

Omnes homines unius artis collegium faciunt, quod paraticum vocant [...].

Hauriunt autem aquam non solum ligneis situlis, set etiam aereis, que sitelle dicuntur, quibus quasi omnes habendant, ministrantes aquam capidibus ereis [...]. Habent etiam sub utroque latere ignis instrumenta ferrea, pluribus necessitatibus apta, que, quia sub igne ponuntur, grece ypopiria, vulgariter autem ibi brandalia nuncupatur (cap. XIII). ${ }^{38}$

37. Cfr. De vulgari eloquentia, cit., p. 76, I.IX.7: «Quapropter audacter testamur quod si vetustissimi Papienses nunc resurgerent, sermone vario vel diverso cum modernis Papiensibus loquerentur».

38. ANONYMI TICINENSIS, Liber de laudibus civitatis Ticinensis, a cura di Rodolfo MAIOCCHI e Ferruccio QUINTAVALLE, Rerum Italicarum Scriptores, XXXIV, Città di Castello: Lapi, 1903; cfr. anche Angelo Stella, Cesare Repossi, Fabio Pusterla, Lombardia, Brescia: La Scuola, 1990, p. 122. 
Raffrontata a quella dell'analogo passo del De magnalibus, la forma Carochium, che nel digramma lascia trasparire il suono palatale, sembra aver acquisito nuova specificità rispetto all'effettiva pronuncia, così come sitella segna rispetto a situlus un'escursione lessicale che in controluce si ipotizza anche fonetica, in un esito che, sulla scorta dei rilievi di Carlo Salvioni, ${ }^{39}$ potrà dirsi tratto distintivo rispetto alla vicina Milano e nella cronaca sinopia per l'etimologia di testi più tardi. ${ }^{40}$

Difficile risulterebbe attraversare la mappa linguistica dell'Italia medievale e ritrovarvi posizione e confini di un volgare, e nello specifico di quelli lombardi, se non venisse in soccorso la venatio del De vulgari eloquentia, che non traccia linee di demarcazione, ma applica i principi di una linguistica areale, fondata sull'ascolto e sulla percezione contrastiva delle zone di influenza.

L'esilio di Dante misura i confini della Lombardia, che si spalanca superata la Marca trevigiana e la Romagna a ovest e sud-ovest, in una sostanziale coincidenza con l'area geolinguistica che Giovan Battista Pellegrini avrebbe assegnato nel 1979 ai dialetti gallo-italici. ${ }^{41}$

Più dei confini precisi, interessa qui il criterio con cui è delimitata la zona lombarda, che affiora, quasi per contrasto rispetto alla dolcezza propria degli Imolesi, quando si ascolta la parlata bolognese:

Accipiunt enim prefati cives ab Ymolensibus lenitatem atque mollitiem, a Ferrarensibus vero et Mutinensibus aliqualem garrulitatem que proprie Lombardorum est: hanc ex commixtione advenarum Longobardorum terrigenis credimus remansisse. ${ }^{42}$

La geografia si intreccia e diviene tutt'uno con la storia, nella lingua come nella politica: lo ricordano all'esule fiorentino i suoi autori, in particolare le etimologie/analogie dei glossari enciclopedici di Isidoro, Papias, Uguccione. Così nella garrulitas dei lombardi/longobardi ${ }^{43}$ si ascolta tutta l'asprezza dell'oltraggio subito:

E quando il dente longobardo morse

la Santa Chiesa, sotto le sue ali

Carlo Magno, vincendo, la soccorse. ${ }^{44}$

39. Carlo SAlvioni, «Dell'antico dialetto pavese», Bollettino della Società pavese di storia patria, 1902, p. 193-251; indicazioni più dettagliate riguardo all'esito localizzante-ELLU(M) > é si ricavano alle p. 201-202.

40. Angelo STELla, Lombardia, cit., p. 155.

41. Giovan Battista Pellegrini, Carta dei dialetti d'Italia, Pisa: Pacini, 1977.

42. Dante Alighieri, De vulgari eloquentia, a cura di Pier Vincenzo Mengaldo, in ID., Opere minori, tomo II, De vulgari eloquentia, Monarchia, Epistole, Egloghe, Questio de aqua et terra, a cura di Pier Vincenzo Mengaldo, Bruno Nardi, Arsenio Furgoni, Giorgio Brugnoli, Enzo Cecchini, Francesco Mazzoni, Milano-Napoli: Ricciardi, 1979, p. 118-121, I.XIV.2-3.

43. Non si dimentichi il rimando a "Pone, sanguis Longobardorum, coaductam barbariem» a indicare i riottosi signori lombardi nell'epistola $V \$ 11$, portato alla luce da Pier Vincenzo Mengaldo. 
L'indicazione è traccia lasciata cadere sul sentiero di un'ipotesi di ricerca e di lettura, che lega Dante agli storici, i cronisti coevi come la generazione di Ludovico Antonio Muratori, impegnati nel tentativo di risolvere il nodo delle invasioni barbariche e degli influssi linguistici delle migrazioni sull'originaria unità della cultura e della lingua, di Roma e d'Italia.

$\mathrm{Al}$ di là di facili ritorni alla «radice» longobarda, l'area su cui si concentra la nostra attenzione può essere definita dall'influsso di elementi germanici, ${ }^{45}$ oggi quasi del tutto assorbiti, su cui molto può dire o suggerire la toponomastica, pur con le riserve che gli storici più attenti sollevano, ${ }^{46}$ e l'onomastica: si consideri nei documenti latini del Codice Diplomatico Bresciano la sopravvivenza di Braida, accanto ai nomi di contraenti e proprietari, tra i quali la già citata badessa Ermengarda, a testimoniare un'epoca prima che una provenienza.

Dalla «silva» dei volgari italiani, dopo l'idioma dei Romani, degli abitanti della Marca Anconitana e degli Spoletini, tocca alla loglio lombardo di venire estirpato:

Post quos Mediolanenses atque Pergameos eorumque finitmos eruncemus, in quorum etiam improperium quendam cecinisse recolimus:

Enter l'ora del vesper, ciò fu del mes d'ochiover. ${ }^{47}$

La localizzazione, quando non passa attraverso il livello «mediocre», esemplifica lo specifico di un'area nell' «improperium»: a tutti gli effetti, in anticipo su Lancino Curti e Luigi Pulci, la citazione dantesca può a buon conto dirsi saggio di un uso "riflesso», che testimonia e stigmatizza i connotati salienti del volgare.

Più ancora dell'esito palatale del nesso latino -CT- (cui non si oppone la grafia -chio-, in linea come visto con le abitudini grafiche dei testi del Duecento), nella citazione dantesca la caduta delle atone finali sembra precludere alla lingua dei milanesi e dei bergamaschi l'accesso al mondo regolato della "gramatica» e del volgare «illustre».

In questa direzione si erano misurati i primi passi del volgare letterario lombardo, nella percezione di una perdita che sempre più era stata percepita come mancanza, ma al tempo stesso come elemento di specificità.

«O gloria d'i Latin», disse, "per cui

mostrò ciò che potea la lingua nostra,

o pregio etterno del loco ond'io fui,

qual merito o qual grazia mi ti mostra». ${ }^{48}$

45. La lista proposta da Claudio BeretTA, Letteratura dialettale milanese, Milano: Hoepli, 2003, p. 14-15, andrebbe forse rivista e letta in chiave storica sulla scorta delle indicazioni di Arrigo CaStellani, Grammatica storica della lingua italiana, vol. I, Introduzione, Bologna: Il Mulino, 2000, in particolare L'elemento germanico, p. 29-94.

46. Aldo A. SeTtIA, Tracce di Medioevo. Toponomastica, archeologia e antichi insediamenti nell'Italia del Nord, Cavallermaggiore: Gribaudo, 1996, in particolare il capitolo I nomi di luogo. Usi e abusi, p. 21-24.

47. De vulgari eloquentia, cit., p. 94-95, I.XI.5.

48. Purgatorio VII 16-19. 
Il personaggio di Sordello, di patria mantovana, esce dal cono d'ombra purgatoriale e si distacca dalla garrulitas lombarda nel segno di quella «lingua nostra» che, superate le invasioni e le cadute, appare unica, continua dal tempo di Virgilio a quello di Dante. «Dolce», come altrove lo «stilo», suonava infatti il nome della città nell'abbraccio dei due mantovani («e l'un l'altro abbracciava» Purgatorio VII 75), nel cuore di quell'invettiva in cui, forse non a caso, i denti rodevano con reminiscenza fonica della torre della Muda e l'eco dell'oltraggio antico di cui si erano macchiati i principi lombardi:

Ahi serva Italia, di dolore ostello,
[...]
e ora in te non stanno senza guerra
li vivi tuoi, e l'un l'altro si rode
di quei ch'un muro e una fossa serra. ${ }^{49}$

La vicenda, celeste e terrena, dell' «anima gentil» riproduce in scala l'esilio di Dante: la vita dell'aldilà, dove Sordello non sembra avere dichiarata collocazione, chiamato com'è ad accompagnare il viaggio, pare a tutti gli effetti specchio ultramondano dell'esilio, che lo aveva fatto vagare di corte in corte, e prefigurazione di un'altra storia terrena. ${ }^{50}$ Ecco allora acquisire profondità la lode affidata al De vulgari eloquentia:

Dicimus ergo quod forte non male opinantur qui Bononienses asserunt pulcriori locutione loquentes, cum ab Ymolensibus, Ferrarensibus et Mutinensibus circunstantibus aliquid proprio vulgari asciscunt, sicut facere quoslibet a finitimis suis conicimus, ut Sordellus de Mantua sua ostendit, Cremone, Brixie atque Verone confini: qui, tantus eloquentie vir existens, non solum in poetando sed quomodocunque loquendo patrium vulgare deseruit. ${ }^{51}$

Il passo, come noto, ha avviato un lungo e non risolto dibattito sui volgari di Sordello, che non sarebbero conservati se non in quel presunto «sermone lombardesco», attribuitogli da Giulio Bertoni, con il successivo placet continiano.

Il corpus superstite delle sue poesie testimonia l'estremo esempio di abbandono del "patrium vulgare» per un provenzale illustre, che, punteggiato degli «italianismi» messi in luce da Marco Boni, ${ }^{52}$ è lasciapassare nell'Italia settentrionale, tra Mantova e la Marca, superando, attraverso uno degli idiomi della «joglaria», mura e fossati delle singole identità culturali.

L'esilio del giullare è il segno, esistenziale e poetico, che ne caratterizza la lingua, e che nella scripta lombarda tra Due e Trecento permette di individuare una

49. Purgatorio VI 76, 82-84.

50. Cfr. La "fortuna" di Sordello fino al secolo XIX, in SORDELLO, Le poesie, nuova edizione critica con studio introduttivo, traduzioni, note e glossario a cura di Marco BonI, Bologna: Libreria antiquaria Palmaverde, 1954, p. CLXXXI-CCII.

51. De vulgari eloquentia, cit., p. 118-121, I, XV, 2.

52. SORDELlO, Le poesie, cit., p. CLXXVI. 
vena attiva e produttiva tra corte e corte, pronta a ritrovare un pubblico, solo diatopicamente eterogeneo, su un terreno comune di parole e di esiti.

Se, come ha ribadito Arrigo Castellani indicando una chiave interpretativa per il Duecento italiano, «i primi passi verso l'unificazione linguistica italiana sono stati fatti sulla strada della poesia lirica», 53 la codificazione del volgare, nei diversi centri di irradiazione di area lombarda, si muove in ambito letterario, dove può e deve misurarsi da subito con i limiti e gli strumenti offerti dal verso.

Almeno inizialmente, e nel codice giullaresco in particolare, la ricerca passa per il conguaglio di elementi, certamente localizzabili, ma comunque fruibili: accanto alla lingua del modello, che agisce da impronta per il calco non solo semantico, e alle marche localizzanti gli editori registrano tracce di venetismi e in generale di quei tratti settentrionali, di recente ravvisati anche nel Ritmo laurenziano. ${ }^{54}$

Parlare di Lombardia, almeno per il Medioevo, significa pensare a un luogo di ricezione e di circolazione di lingue, che non funzionano come agenti normalizzanti, ma come canale sistematicamente percorso e attivo per la poesia lirica. Solo con il tardo Duecento, la coesistenza di elementi di diversa provenienza geografica andrà ad allinearsi sotto il verticale potere di attrazione della grammatica toscana, precocemente messa a punto dai poeti menzionati nel De vulgari eloquentia.

La ricerca di un codice linguistico comune, che superasse le divisioni territoriali e politiche, sembra governare la tensione interna all'area lombarda, per cui definire le norme del volgare, e in particolare le tendenze grafiche, significa ritrovare tratti e tensioni condivise da una "grammatica" che alle origini risulta sostanzialmente complementare al latino.

In questa direzione si muovono gli inizi volgari, che in area lombarda non sono affidati ai libri di conti o alle iscrizioni, come è di altre regioni e in particolare della Toscana medievale, ma a testi letterari, ${ }^{55}$ primo in ordine di tempo e di riflessi seriori il manoscritto Saibante-Hamilton 390, conservato alla Deutsche Staatsbibliothek di Berlino, che ha tramandato, pochi decenni dopo la composizione, il Libro di Uguçon de Laodho ${ }^{56}$ e lo Splanamento de li Proverbii de Salamone "composto per Girardo Pateg da Cremona». 57

L'affioramento grafico del volgare in Lombardia si attesta in quell'area del documento, come della vita e della lingua, descritta già dai documenti latini, in cui è possibile e deve, ormai necessariamente, avvenire la mediazione tra due culture linguistiche, non più affidata di volta in volta a un funzionario, ma una volta per tutte incarnata dall'autore.

53. Arrigo CASTELLANI, Grammatica storica della lingua italiana, vol. I, Introduzione, cit., in particolare il cap. VI, Cenni sulla formazione della lingua poetica, p. 459-536; la citazione è a p. 462.

54. Ibid., p. 465-466.

55. Sulla questione cfr. Paolo Bongrani, Silvia Morgana, «La Lombardia», in Francesco BRUNI (a cura di), L'Italiano nelle regioni. Lingua nazionale e identità regionali, Torino: UTET, 1992, p. 84-142, in particolare Le prime testimonianze del volgare in Lombardia, p. 87-95.

56. Gianfranco ConTini, Poeti del Duecento, Milano-Napoli: Ricciardi, 1960, t. I, p. 597-624.

57. Ibid., p. 557-588. 
Se i primi testi fiorentini si muovono sull'asse orizzontale della famiglia, di cui le "organizzazioni affaristiche che sorgono nei secoli XII e XIII non sono che estensioni e perfezionamenti», ${ }^{8}$ con l'agilità e la naturalezza di un "dialetto» che è linguaggio specialistico quando ormai non gergo, le attestazioni dei volgari di area lombarda sembrano collocarsi sull'asse verticale di un allontanamento dai tratti municipali e dell'adesione a una dottrina, semplificata e esemplificata, da trasmettere a nuovi ascoltatori.

In Lombardia i primi passi del volgare si muovono nella direzione del volgarizzamento, dell'esplanamento di una cultura che doveva «trovare» parole ormai mutate per i medesimi referenti, non diversamente da quanto la cultura e il lessico scolastici suggerivano a chi apparecchiava il "generale convivio». 59

Ecco ben chiara fin dai primi distici di Gerardo Patecchio la distinzione tra «li savi [...] q’ig sa ben çò q'ig dé» e quei "comunal omini, qe no san ogna le’" (v. 13-16), questi ultimi destinatari dell'opera, non tanto per l'affacciarsi di un nuovo pubblico alla vita politica e culturale, ma per la scelta consapevole del mediatore di una lingua, con compiti analoghi e ben più vasti rispetto a quelli svolti da Otto notarius e dai suoi colleghi, di coinvolgere un'intera società intorno a un codice morale comune, a identificare una categoria di lettori e di uomini "cui illi vol si sia", ma pronti ad ascoltare e a mettere a frutto il messaggio («se tuto 'l ben adovra / q'eu voi dir e 'l mal lassa, no pò far meior ovra» v. 17-18).

Di qui passa, in anni non lontani, la ricerca di uno stile che appartenga a una categoria di lettori e a un pubblico, che, di là del Po e del Reno, avrà per qualità e segno distintivo gentileça e cortesia. Comune ha da essere anche il mezzo, oltre che il pubblico: entrambi vanno preparati, assemblati, anche là dove la tendenza di ciascuna varietà a dimostrarsi locale e l'unicità di ciascun contesto cittadino rimangono accentuate.

Il codice Saibante-Hamilton, come provato dai rilievi fonico-metrici di Gianfranco Contini, fotografa una fase arcaica del volgare di area lombarda, in cui è però già evidente la tendenza alla caduta delle finali diverse da $a$, decisamente conclusa all'epoca in cui Dante registra il primo verso dell' «improperium» e il copista del codice Berlinese Ital. qu. 26 della Staatsbibliothek di Berlino trascrive i volgari di Bonvesin.

Non diverso da quello di "Cynus Pistoriensis et amicus eius» ${ }^{60}$ è il tentativo di Gerardo, o più probabilmente del copista, di appoggiarsi a una gramati$c a$, che non è soltanto quella del latino (come vorrebbe il ripristino grafico di $\mathrm{AU}$, anche quando non latino, e dei nessi consonantici con L, segnalati da Contini), ma terreno espressivo in cui erano confluiti elementi grafico-fonetici

58. Arrigo Castellani, "Carattere dei testi. Cenni sui libri del dare e dell'avere», in Nuovi testi fiorentini del Dugento, con introduzione, trattazione linguistica e glossario a cura di Arrigo CASTEllani, Firenze: Sansoni, 1952, t. I, p. 2-10; la citazione è a p. 2.

59. Convivio, cit., p. 10, I.I.11.

60. De vulgari eloquentia, p. 84-87, I.x.2: "primo quidem quod qui dulcius subtiliusque poetati vulgariter sunt, hii familiares et domestici sui sunt, puta Cynus Pistoriensis et amicus eius; secundo quia magis videntur initi gramatice que comunis est, quod rationabiliter inspicientibus videtur gravissimum argumentum». 
codificati dagli antecedenti di area contigua, in primo luogo da quei Proverbia que dicuntur super natura feminarum, tramandati proprio dal SaibanteHamilton: le marche venete (forse attribuibili al copista), -mentre per gli avverbi modali e la presenza dei dittonghi da $e$ aperta, ${ }^{61}$ i tratti di una lenizione che accompagnerà i primi passi del volgare (marcata dal digramma - $d h-)$, a segnare anche nella grafia una linea di continuità, che andrà gradualmente dissolvendosi nei manoscritti di Bonvesin de la Riva.

Ecco però che proprio la maggiore solidità delle vocali nel Libro di Uguccione da Lodi fa propendere per una datazione del testo in epoca precedente rispetto a Patecchio, fase intermedia rispetto a quella segnata dalla trascrizione dei Proverbia. L'originaria resistenza delle vocali finali e in genere delle atone, residuo della semplificazione naturale rispetto alla gramatica, resta tratto grafico caratterizzante una scripta che vuole salvare, ancora nel tardo Duecento, l'appoggio di una lingua di riferimento, che sta lentamente cambiando referente.

Almeno agli esordi dell'esperienza volgare, il sistema grafico, costruito sul latino, non doveva apparire insufficiente, come oggi può sembrare a chi affronti il problema della resa delle vocali turbate in area gallo-italica: in mancanza di un "adeguato simbolo», il copista del codice berlinese di Bonvesin trovava ad esempio in $o$ il «solo possibile spediente» ${ }^{62}$ per rendere la $\ddot{o}$, dove si sarebbe ravvisato l'«imporsi della lettera al suono». ${ }^{63}$

Interrogare le scelte poetiche degli autori significa in prima istanza misurare e ammettere le variazioni dei singoli copisti, affidarsi alla storicità della loro lingua, evitando seriori interpolazioni e ricostruzioni fondate sul principio dell'autonomia del «dialetto». La ricerca dell'originale parola dell'autore, ormai nelle linee e negli strumenti ricostruita dalla silloge ricciardiana e dalle CLPIO, andrebbe forse letta dal rovescio dell'arazzo, interpretando l'intervento normalizzante di ciascun copista, come tentativo di attualizzare e al contempo di eternare la lingua del poeta e dello scrittore.

La rima core: amore, e in generale la consonanza di ó con ò, comune ai testi settentrionali e siciliani, accanto ad altri indizi fonetici, ha suggerito la presenza di "un "sistema unitario" anteriore ai più antichi testi volgari della nostra letteratura in versi», ${ }^{64}$ una sorta di koinè che precede e che fonda in aree diverse la grammatica della lingua poetica.

61. Cfr. Piera Tomasoni, "Veneto», in Luca Serianni e Pietro Trifone (a cura di), Storia della lingua italiana, vol. III, Le altre lingue, cit., p. 212-240.

62. Carlo SALVIONI, "Osservazioni sull'antico vocalismo milanese desunte dal metro e dalla rima del cod. Berlinese di Bonvesin da Riva», in Studi Letterari e Linguistici dedicati a Pio Rajna nel quarantesimo anno del suo insegnamento, Milano: Hoepli, 1911, p. 367-388, la citazione è a p. 369.

63. ID., Fonetica del dialetto moderno della città di Milano, Roma-Torino-Firenze: Loescher, 1884, Prefazione, p. 9.

64. Concordanze della lingua poetica italiana delle origini (CLPIO), vol. I, a cura di D'Arco Silvio Avalle e con il concorso dell'Accademia della Crusca, Milano-Napoli: Ricciardi, 1992, p. CCXXIX-CCXXX. 
Alla luce di questa e di altre consonanze, di cui il Saibante-Hamilton e il berlinese di Bonvesin, come i testimoni toscani, si fanno portavoce, D’Arco Silvio Avalle poteva concludere che nella seconda metà del Duecento, sulla base omogenea di ricerca di un codice poetico, si sarebbe fatta avanti la Toscana, con un ruolo di mediazione tra le spinte del Nord e quelle del Sud, «armonizzandole in una spinta superiore».

Lo sforzo unitario che verrà poi monopolizzato dalla cultura fiorentina attraverso una lenta opera di decantazione delle scorie «municipali» (si veda il caso di Dante), è il primo serio tentativo di fondare una grammatica generale (se non altro competitiva nei confronti di quella del latino) da estendersi ad una vasta area geopolitica ancora priva di un serio centro di coagulo linguistico. ${ }^{65}$

Punto di contatto e di frizione, oltre che membrana osmotica, la grafia, intesa nella sua complessità di strumento concreto di trasmissione, testimonia questo sforzo, mezzo e al contempo confine su cui sarà dato di misurare anche il rapporto tra la lingua e il dialetto. 